\title{
La Vita S. Pauli Monachi Thebaei di san Girolamo contenuta nel codice manoscritto Vaticanus Latinus 13008
}

Nell'articolo presentiamo il testo della Vita S. Pauli Monachi Thebaei di san Girolamo, contenuto nel codice manoscritto Vaticanus Latinus 13008 e custodito presso la Biblioteca Apostolica Vaticana. Esponiamo questo testo dell'opera geronimiana, perché il manoscrit to che lo contiene non era noto né a John Frank Cherf, O.S.B. ${ }^{2}$, né fu usato per la nostra edizione critica della Vita S. Pauli ${ }^{3}$, e questo per il semplice fatto che esso entrò a far parte della raccolta dei codici manoscritti della Biblioteca Apostolica Vaticana soltanto nell'anno $1924^{4}$. Per tale ragione, il Vaticanus Latinus 13008 non appare nel catalogo dei codici agiografici latini, conservati pres-

1 Prof. dr. hab. Bazyli Degórski O.S.P.P.E., professore di patrologia e di teologia dogmatica presso la Facoltà di Teologia della Pontificia Università S. Tommaso d'Aquino a Roma, e professore di patrologia e di antropologia patristica presso la Pontificia Facoltà Teologica “Teresianum” a Roma; e-mail: osppe.roma@gmail.com; ORCID 0000-0003-1997-2769.

2 Cf. J.F. Cherf, The Latin Manuscript Tradition of the "Vita Sancti Pauli", in: Studies in the Text Tradition of. St. Jerome's "Vitae Patrum”, ed. W.A. Oldfather, Urbana 1943, p. 133-142.

3 Cf. B. Degórski, Edizione critica della "Vita Sancti Pauli Primi Eremitae" di Girolamo, Institutum Patristicum "Augustinianum”, Roma 1987. Non abbiamo analizzato il Vaticanus Latinus 13008 neanche nelle altre nostre pubblicazioni concernenti i manoscritti vaticani che contengono l'opera geronimiana in questione. Cf. B. Degórski, Przekaz łacińskich rękopisów Vita Sancti Pauli Primi Eremitae św. Hieronima, Lublin 2000; B. Degórski, I manoscritti della Vita Sancti Pauli Primi Eremitae di san Girolamo conservati nella Biblioteca Apostolica Vaticana, Lublin 2005; B. Degórski, I manoscritti della Vita Sancti Pauli Primi Eremitae di San Girolamo, conservati nelle biblioteche di Roma, che contengono testo incompleto dell'opera geronimiana o che si ispirano ad essa, Lublin 2011.

4 J. Leclercq, S. Antoine dans la tradition monastique médiévale, "Studia Anselmiana" 38 (1956) p. 246. 
so la Biblioteca Apostolica Vaticana, pubblicato nel 1910 dal gesuita padre Albertus Poncelet ${ }^{5}$. Sembra, quindi, molto utile e opportuno fornire il testo della Vita S. Pauli Monachi Thebaei, tramandato a noi da questo antico codice.

\section{Descrizione del codice}

Il codice Vaticanus Latinus 13008 proviene dall'XI secolo. La sua scrittura è una bella e regolare carolingia. Le dimensioni del codice sono le seguenti: $32 \times 21 \mathrm{~cm}$. Il manoscritto contiene 244 fogli di cartapecora ${ }^{6}$, scritti a due colonne.

La Vita S. Pauli Monachi Thebaei comincia al foglio $187 \mathrm{v}$ nel seguente modo: [in rosso:] Incipit uita $S[$ ancti].P[auli]. Heremite ędita a beato hieronimo $p[$ res $] b[y t e]$ ro. Inter multos sepe dubitatum est $[\ldots]$, e finisce al foglio 192 con le parole: [...] quam regum purpuram cum regnis suis. Quindi, senza alcuna aggiunta dello scrivano.

Il testo della Vita S. Pauli Monachi Thebaei viene diviso in dodici capitoli, nel seguente modo: capitolo I: Inter multos [...] quam ab eo omnium incitata sunt studia; capitolo II: Amathas uero \& macharius [...] referenda sententia quidem uidetur; capitolo III: Igitur quia de antonio [...] due memoriae causa, exempla subicimus; capitolo IV: Perseuerantem in fide martyrem [...] succedens doloris magnitudo superauit; capitolo V: Per idem ergo tempus [...] crudelitate quasi pietate utebatur; capitolo VI: Quod ubi prudentissimus adolescens intellexit [...] ut ab imperatore uideretur allatum est; capitolo VII: Sed ut propositum persequar [...] gratiae d[omi] no in communae referuntur; capitolo VIII: Et ut post sanctum osculum [...] meror suae mortis leuaretur; capitolo IX: Stupefactus ergo antonius [...] ad monasterium quod postea a sarracenis occupatum est, regrediebatur; capitolo X: Neque uero gressus [...] ut ad instar auis peruolaret; capitolo XI: Nec immerito [...] semper pauli tunicam uestitus est; capitolo XII: Libet in fine opusculi [...] quam regum purpuram cum regnis suis.

5 Cf. A. Poncelet, Catalogus codicum hagiographicorum Latinorum Bibliothecae Vaticanae, Subsidia hagiographica 11, Bruxelles 1910.

6 Di questo codice parla anche M.E. Lage Cotos, Sobre los "abusiva” de Huesca, "Studi medievali" 36 (1995) p. 937. 


\section{Il metodo adoperato per la presentazione del codice}

Pubblicando il testo dell'opera geronimiana, contenuto nel manoscritto Vaticanus Latinus 13008, abbiamo adoperato i seguenti principi: per poter cogliere le diverse varianti del testo geronimiano, nella colonna sinistra abbiamo fornito il testo dell'edizione critica della Vita Sancti Pauli Primi $E_{\text {Eremitae }}{ }^{7}$, in quella, invece, che si trova a destra, abbiamo collocato il testo del manoscritto preso in esame. Ogni lezione o variante che differisce nei due testi è stata contraddistinta, in ambo le colonne, dal grassetto. Inoltre, nelle note esplicative abbiamo aggiunto tutte le spiegazioni riguardanti sia le correzioni che le abrasioni, nonché delle altre notizie ritenute importanti per potersi rendere conto del testo del manoscritto analizzato.

\section{Il testo della Vita S. Pauli Monachi Thebaei di san Girolamo}

\section{Degórski}

Inter multos saepe dubitatum est a quo potissimum monachorum eremus habitari coepta sit.

Quidam enim altius repetentes, a beato Elia et Ioanne principia sumpserunt. Quorum et Elias plus nobis uidetur fuisse, quam monachus, et Ioannes ante prophetare coepisse quam natus est.

Alii autem, in quam opinionem uulgus omne consentit, adserunt Antonium huius propositi caput, quod ex parte uerum est. Non enim tam ipse ante omnes fuit, quam ab eo omnium incitata sunt studia.

Amatas uero et Macarius, discipuli Antonii, e quibus superior corpus magistri sepeliuit, etiam nunc adfirmant, Paulum quemdam Thebaeum principem rei istius

\section{Il codice Vaticanus Latinus 13008}

[f. $187 \mathrm{v}$ ] Inter multos sepe dubitatum est, a quo potisimum monachorum heremus habitari cepta sit.

Quidam enim altius repetentes, a beato helia et iohanne principia sumpserunt, quorum et helias plus nobis uidetur fuisse quam monachus, et iohannes ante prophetare cepise ${ }^{8}$ quam natus est ${ }^{9}$.

Alii autem, in quam opinionem uulgus omne consentit, asserunt antonium, huius propositi caput, quod ex parte uerum est. Non enim tam ipse ante omnes fuit, quam ab eo omnium incitata sunt studia.

Amathas uero et Macharius discipuli antonii, e quibus superior corpus magistri sepeliuit, etiam nunc affirmant, paulum quendam thebeum principem rei istius

\footnotetext{
Cf. Degórski, Edizione critica, passim.

Certo: cepise*****.

9 Certo: $\mathrm{e}^{*}$. Lectio ante correctionem: esset, ut videtur.
} 
fuisse, non nominis, quam opinionem nos quoque probamus. Nonnulli et haec et alia, prout uoluntas tulit, iactitant: subterraneo specu crinitum calcaneo tenus hominem, et multa quae persequi otiosum est incredibilia fingentes. Quorum quia impudens mendacium fuit, ne refellenda quidem sententia uidetur. Igitur quia de Antonio tam Graeco quam Romano stilo diligenter memoriae traditum est, pauca de Pauli principio et fine scribere disposui; magis quia res omissa erat, quam fretus ingenio. Quomodo autem in media aetate uixerit, aut quas Satanae pertulerit insidias, nulli hominum compertum habetur. Sub Decio et Valeriano persecutoribus, quo tempore Cornelius Romae, Cyprianus Carthagine felici cruore damnati sunt, multas apud Aegyptum et Thebaidem Ecclesias tempestas saeua populata est.

Voti tunc Christianis erat, pro eo nomine gladio percuti. Verum hostis callidus tarda ad mortem supplicia conquirens animas cupiebat iugulare, non corpora. Et ut ipse, qui ab eo passus est, Cyprianus ait: Volentibus mori non permittebatur occidi. Cuius ut crudelitas notior fiat, duo memoriae causa exempla subiecimus. Perseuerantem in fide martyrem, et inter eculeum laminasque uictorem, iussit melle perungi, et sub ardentissimo sole, religatis post tergum manibus, reponi, scilicet ut muscarum aculeis cederet, qui ignitas sartagines ante superasset.

Alium iuuenali aetate florentem in amoenissimos hortulos praecepit adduci; fuise ${ }^{10}$, non nominis, quam opinionem nos quoque probamus. Non nulli haec et alia prout uoluntas tulit, iactitant sub terraneo specu crinitum calcaneotenus hominem, et multa quae persequi otiosum est, incredibilia fingentes. Quorum quia impudens mendacium fuit, nec referenda sententia quidem $^{11}$ uidetur. Igitur quia de antonio tam greco quam romano stilo, diligenter memoriae traditum est, pauca de pauli principio et fine scribere disposui; magis quia res omissa erat, quam fretus ingenio. Quomodo autem in media etate uixerit, aut quas satanae pertulerit insidias, nulli hominum compertum habetur. Sub decio et ualeriano persecutoribus, quo tempore cornelius romae, cyprianus cartagine ${ }^{12} \mathrm{fe}-$ lici cruore [f. 188] dampnati sunt, multo apud aegyptum et thebaidem, tempestas seua populata est.

Uoti tunc xpianis erat, pro nomine xpi gladio percuti. Verum hostis callidus, tarda ad mortem supplicia conquirens, animas cupiebat iugulare, non corpora. Et ut ipse qui abeo pasus ${ }^{13}$ est, Cyprianus ait:

Uolentibus mori, non permittebatur occidi. Cuius ut crudelitas notior fiat, duo memoriae causa, exempla subicimus. Perseuerantem in fide martyrem, et inter eculeos flammasque uictorem, iussit melle perungi ${ }^{14}$, et sub ardentissimo sole, ligatis posttergum manibus reponi, scilicet ut muscarum aculeis cederet, qui ignita sartagine ${ }^{15}$ ante superasset.

Alium iuuenili aetate florentem, in amęnisimo hortulo ${ }^{16}$ precepit adduci; ibique

10 Secunda manus in fuisse correxit.

11 Secunda manus in quidem sententia correxit.

12 Secunda manu; prima manu cartaginae, ut videtur.

13 Certo: **passus (ante correctionem: cōpassus, ut videtur); secunda manus in passus correxit.

14 Certo: $\mathrm{p}$ [er]ungi*.

15 Secunda manus in ignitas sartagines correxit.

16 Secunda manus in amęnissimos Hortulos correxit. 
ibique inter candentia lilia et rubentes rosas, cum leni iuxta murmure serperet riuus, et molli sibilo arborum folia uentus stringeret, super structum plumis lectulum supinari, et ne se inde posset excutere, blandis sertorum nexibus inretitum relinqui.

Quo cum, recedentibus cunctis, meretrix speciosa uenisset, coepit delicatis stringere colla complexibus, et, quod dictu quoque scelus est, manibus adtrectare uirilia, ut corpore in libidinem concitato, se uictrix impudica superiaceret. Quid ageret miles Christi, quo se conferret? Quem tormenta non uicerant, superabat uoluptas.

Tandem coelitus inspiratus, praecisam mordicus linguam in osculantis se faciem exspuit; ac sic libidinis sensum succedens doloris magnitudo calcauit.

Per idem ergo tempus, quo talia gerebantur, apud inferiorem Thebaidem, cum sorore iam uiro tradita, morte amborum parentum, in haereditate locupleti Paulus relictus est, annorum circiter sexdecim, litteris tam Graecis quam Aegyptiacis adprime eruditus, mansueti animi, Deum ualde amans. Et dum persecutionis detonaret procella, in uilla remotiore secretior erat. Verum quid pectora humana non cogis, auri sacra fames? Sororis maritus coepit prodere uelle, quem celare debuerat.

Non illum uxoris lacrimae, non communio sanguinis, non exspectans cuncta ex alto inter candentia lilia, ac rubentes rosas, cum leni iuxta murmure serperet riuus, et molli ${ }^{17}$ sibilo arborum folia uentus stringeret,

super stratum plumis $^{18}$ lectum ${ }^{19}$ supinari, blandis sertorum nexibus irretitum relinqui.

Quo $^{20}$ cum recedentibus cunctis, meretrix speciosa ueniset ${ }^{21}$, caepit delicatis constringere colla complexibus. Et quod dictu quoque scelus est, manibus attrectare uirilia, ut corpore in libidinem concitato, se uictrix impudica super iaceret. Quid ageret miles xi? quo se conferret? quem tormenta non uicerant, superaret ${ }^{22}$ uoluptas.

Tandem caelitus inspiratus, $\mathrm{p}$ [rae]cisa[m] mordicus linguam, in osculantis faciem expulit $^{23}$; ac sic libidinis sensum, succedens doloris magnitudo ${ }^{24}$ superauit.

Per idem ergo tempus quo talia gerebantur, apud inferiorem thebaidam, cum sorore sua iam uiro tradita, morte amborum parentum, in hereditate locupleti, paulus relictus est annorum circiter sedecim, litteris tam grecis, quam aegyptiacis [f. $188 \mathrm{v}$ ] aprime eruditus, mansueti animi, deum ualde amans, et dum persecutionis detonaret procella, in uilla remotiore secretior fuit. Verum, quid pectora humana non cogis auri sacra fames? Sororis maritus, caepit prodere uelle, quem celare debuerat.

Non illum ut assolet uxoris lacrimae, non communio sanguinis, non spectans exalto

\footnotetext{
17 I secunda manu, ut videtur, super rasuram.

18 Certo: plu*mis (i super rasuram, s super lineam).

19 Secunda manus, ut videtur, in margine dextero lectvlū scripsit.

20 Quo secunda manu, ut videtur, super rasuram.

21 Secunda manus in uenisset correxit.

22 Re secunda manu, ut videtur, super rasuram. Lectio ante correctionem: supera-

23 Secunda manus in expuit correxit.

24 O secunda manu super rasuram.
} bat, $u$ t videtur. 
Deus, a scelere reuocauerunt. Aderat, instabat, crudelitate quasi pietate utebatur. Quod ubi prudentissimus adulescens intellexit, ad montium deserta confugiens, dum persecutionis finem praestolatur, necessitatem in uoluntatem uertit, ac paulatim procedens, rursusque tantumdem, atque idem saepius faciens, repperit saxeum montem, ad cuius radices haud grandis spelunca lapide claudebatur, quo remoto, ut est cupiditas hominum auidius occulta cognoscere, animaduertit intus grande uestibulum, quod aperto desuper coelo, patulis diffusa ramis uetus palma contexerat, fontem lucidissimum ostendens, tantummodo foras statim eadem, quae genuerat, terra sorbebat.

Erant praeterea per exesum montem haud pauca habitacula, in quibus scabrae iam incudes et mallei, quibus pecunia signatur, uisebantur. Hunc locum Aegyptiorum litterae ferunt furtiuam monetae officinam fuisse, ea tempestate qua Cleopatrae iunctus Antonius est.

Igitur adamato B quasi a Deo sibi offerretur B habitaculo, omnem ibidem orationibus et solitudine duxit aetatem. Cibum et uestimentum palma praebebat. Quod ne cui impossibile uideatur, Iesum testor et sanctos angelos eius in ea parte eremi, quae iuxta Syriam Saracenis iungitur, et uidisse me monachos, et uidere, e quibus unus triginta iam per annos clausus hordeaceo pane et lutulenta aqua uiuit. deus a scelere reuocauit, ad erat, instabat, crudelitate quasi pietate utebatur.

Quod $^{25}$ ubi prudens adolescens intellexit, ad montium deserta confugiens, dum persecutionis finem $\mathrm{p}$ [rae]stolat[ur], necesitatem $^{26}$ in uoluntatem uertit, ac paulatim procedens, rursusque tantumdem subsistens, atque idem sepius faciens, repperit saxeum montem, ad cuius radices non ${ }^{27}$ grandis spelunca lapide claudebatur. Quo remoto, ut est cupiditas hominum, auidius occulta cognoscere, animaduertit intus grande uestibulum, quod aperto desuper caelo, patulis diffusa ramis, uetus palma contexerat, fontem lucidissimum ostendens, cuius riuum tantummodo foras, eadem, q[uae] genuerat terra sorbebat. Erant preterea per excisum montem, aut ${ }^{28}$ pauca habitacula, in quibus $\mathrm{scabrae}^{29}$ incudes, et mallei quibus pecunia signabatur uisebantur. Hunc locum aegyptiorum litterae, furtiuam monetae officinam fuisse, ea tempestate, qua cleopate ${ }^{30}$ iunctus antonius est.

Igitur ad amatum quasi a deo sibi offerretur habitaculum, omnem ibi orationibus et solitudine, duxit aetatem. Cibum et uestimentum, palma $\mathrm{p}[\mathrm{rae}]$ bebat, quod ne cui impossibile uideatur, ihm testor et sanctos angelos eius, in hac parte heremi, quae iuxta syriam saracenis iungitur, et uidisse me monachos et uidere, e quibus unum xxx iam per annos clausum, ordeacium $^{31}$ panem, et lutulentam aquam bibentem.

25 Certo: Quod ***.

26 Secunda manus, ut videtur, in necessitatem correxit.

27 Secunda manus in margine sinistro haud scripsit.

28 Secunda manus in haud correxit.

29 Post scabrae secunda manus in margine sinistro iam addidit.

30 Sic! Certo: cleo secunda manu super rasuram; ultima e prima manu, ut videtur, super lineam.

31 Secunda manus in hordeacium correxit (h super lineam scripta est). 
Alter in cisterna ueteri (quam gentili sermone Syri gubbam uocant) quinque caricis per singulos dies sustentatur.

Haec incredibilia uidebuntur eis, qui non crediderint omnia possibilia esse credentibus. Sed ut ad id redeam unde digressus sum, cum iam centesimo tertio decimo aetatis suae anno beatus Paulus coelestem uitam ageret in terris, et nonagenarius in alia solitudine Antonius moraretur, ut ipse adserere solebat, haec in mentem eius cogitatio incidit, nullum ultra se monachorum in eremo consedisse.

Atque illi per noctem quiescenti reuelatum est, esse alium interius multo se meliorem, ad quem uisendum properare deberet.

Illico erumpente luce, uenerabilis senex infirmos artus baculo regente sustentans, coepit ire uelle quo nesciebat. Et iam media dies coquente desuper sole feruebat, nec tamen a coepto itinere deducebatur dicens: Credo in Deo meo, quod seruum suum, quem mihi promisit, ostendet. Nec plura his, conspicatur hominem equo mixtum, cui opinio poetarum Hippocentauro uocabulum indidit. Quo uiso, salutaris impressione signi armat frontem, et heus tu, inquit, quanam in parte Dei seruus hic habitat?

At ille barbarum nescio quid infrendens, et frangens potius uerba quam proloquens inter horrentia ora, satis blandum quaesiuit adloquium. Et cum dexterae manus protensione cupitum indicat iter, ac sic patentes campos uolucri transmittens fuga, ex oculis mirantis euanuit. Verum hoc utrum diabolus ad terrendum eum simulauerit, an, ut solet, eremus monstruosorum ferax animalium istam quoque gignat bestiam, incertum habemus.
Alterum in cisterna ueteri, quam gentili sermone syricumbam uocant, quinque caricis per singulos dies sustentatum. Haec incredibilia uidebuntur [f. 189] eis, qui non crediderint, omnia possibilia esse credentibus. Sed ut ad id regrediar, unde digressus sum, cum iam centesimo tertio decimo aetatis suae anno, beatus paulus caelestem uitam ageret in terris, ac nonagenarius in alia solitudine antonius moraretur, ut ipse asserere solebat, haec in mentem eius cogitatio incidit, nullum ultra se monachorum in heremo consedisse. Atque illi per noctem quiescenti reuelatum est, esse alium interius multum se meliorem, ad quem uisendum deberet properare.

Ilico erumpente luce, uenerabilis senex infirmos artus baculo regente sustentans, caepit ire uelle quo nesciebat. Et iam media dies, coquente desuper sole feruebat, nec tamen a cepto ${ }^{32}$ itinere deducebatur dicens: Credo in deo meo, quia seruum suum quem mihi promisit, ostendet. Nec plura$\mathbf{a s}^{33}$ conspicatur hominem, equo mixtum, cui opinio poetarum centauri ${ }^{34}$ uocabulum indidit. Quo uiso, salutaris inpressione signi armat frontem, heus tu? inquit, quanam in parte, dei seruus hic habitat?

Ille barbarus, nescio quid frendens, et frangens potius uerba quam proloquens, inter horrentia ora seuientis, blandum quaesiuit alloquium. Et cum dexterae manus protensione, cupitum indicat iter, \& sic patentes campos, uolucri transmittens fuga, ex oculis mirantis euanuit. Verum hoc utrum diabolus ad terrendum eum simulauerit, an ut solet heremus monstruosorum ferax animalium, istam quoque gignat bestiam, incertum habetur.

32 Certo: a*cepto (lectio ante correctionem: accepto).

33 Secunda manus in plura his correxit.

$34 \mathrm{U}$ secunda manu, ut videtur, super lineam. 
Stupens itaque Antonius, et de eo quod uiderat secum uoluens, ulterius progrediebatur. Nec mora, inter saxosam conuallem haud grandem homunculum uidet, aduncis naribus, fronte cornibus asperata, cuius extrema pars corporis in caprarum pedes desinebat. Et hoc adtonitus exspectaculo, scutum fidei et loricam spei bonus proeliator arripuit.

Nihilominus memoratum animal palmarum fructus ad uiaticum, quasi pacis obsides, offerebat. Quo cognito, gradum pressit Antonius, et quisnam esset interrogans, hoc ab eo responsum accepit: "Mortalis ego sum, et unus ex accolis eremi, quos uario delusa errore gentilitas, Faunos, Satyrosque et Incubos colit. Legatione fungor gregis mei. Precamur ut pro nobis communem Dominum depreceris; salutem mundi olim uenisse cognouimus, et in uniuersam terram exiit sonus eius".

Talia eo loquente, longaeuus uiator ubertim faciem lacrimis rigabat, quas magnitudo laetitiae indices cordis effuderat.

Gaudebat quippe de Christi gloria, de interitu Satanae: simulque admirans quod eius possit intellegere sermonem, et baculo humum percutiens, aiebat: "Vae tibi, Alexandria, quae pro Deo portenta ueneraris. Vae tibi, ciuitas meretrix, in qua totius orbis daemonia confluxere. Quid nunc dictura es?:

Bestiae Christum loquuntur"!

Necdum uerba compleuerat, et quasi pennigero uolatu petulcum animal aufugit. Hoc ne cui ad incredulitatem scrupulum moueat, sub rege Constantio, uniuerso mundo teste, defenditur. Nam Alexan-
Stupens itaque antonius, et de eo quod uiderat secum uoluens, ulterius progrediebatur. Nec mora inter saxosum ${ }^{35}$ conuallem, non grandem homunculum uidet, aduncis naribus, fronte cornibus asperata, cuius extrema pars corporis, in caprarum pedes desinebat. Et hoc antonius spectaculum, scuto fidei, et lorica spei, [f. 189 $\mathrm{v}$ ] fortis $\mathrm{p}$ [roe]liato[r] arripuit, nichilominus memoratum animal palmarum fructus, ad uiaticum quasi pacis obsides offerebat, quo cognito, gradum pressit antonius, et quis nam esset interrogans, hoc $a b$ eo responsum accepit: mortalis ego sum, et unus ex accolis heremi, quos uarios ${ }^{36}$ delusa errore gentilitas, faunos, satyrosque et incubos colit. Legatione fungor gregis mei, precamur ut pro nobis communem dominum preceris; quem pro salute mundi olim uenisse cognouimus, et in uniuersam terram exiit sonus eius.

Talia eo loquente, longeuus uiator ubertim faciem lacrimis Rigabat, quas magnitudo laetitiae, indices effuderat.

Gaudebat quippe de xpi gloria, de interitu sathanae: simulque ammirans, quod eius posset intellegere ${ }^{37}$ sermonem, et bacillo humum percutiens aiebat: Vae tibi alexandria, quae pro deo portenta ueneraris. Vae tibi ciuitas meretrix, in qua totius orbis daemonia confluxere. Quid nunc dictura es?:

bestiae xpm loquuntur, \& tu pro deo portenta ueneraris?

Nec dum uerba compleuerat, et quasi pennigero uolatu, petulcum animal aufugit. Hoc ne cui ad incredulitatem scrupulum moueat, sub rege constantio, uniuerso mundo teste defenditur. Nam alexandriae

35 Secunda manus correxit. svm secunda manu super lineam. Secunda manus saxosū etiam in margine dextero scripsit.

36 S secunda manus, ut videtur, delevit.

37 Secunda manus in intelligere correxit. 
driam istiusmodi homo uiuus perductus magnum populo spectaculum praebuit, et postea cadauer exanime, ne calore aestatis dissiparetur, sale infusum, et Antiochiam, ut ab imperatore uideretur, adlatum est.

Sed ut propositum persequar, Antonius coepta regione pergebat, ferarum tantum uestigia intuens, et eremi latam uastitatem. Quid ageret, quo uerteret gradum? Iam altera fluxerat dies, restabat unum, ut deseri se a Christo non posse confideret. Pernox secundas in oratione exigit tenebras, et dubia adhuc luce, haud procul intuetur lupam sitis ardoribus anhelantem, ad radices montis inrepere.

Quam secutus oculis, et iuxta speluncam, cum fera abiisset, accedens, coepit introspicere, nihil curiositate proficiente, tenebris arcentibus uisum. Verum, ut scriptura ait: "Perfecta dilectio foras mittit timorem", suspenso gradu et anhelitu temperato, callidus explorator ingressus est, ac paulatim progrediens saepiusque subsistens, sonum aure captabat. Tandem per caecae noctis horrorem procul lumen intuitus, dum auidius properat, offensum pede lapidem in strepitum concitauit. Post cuius sonitum beatus Paulus ostium, quod patebat, recludens, sera obfirmauit. Tunc uero Antonius pro foribus conruens, usque ad sextam et eo amplius horam, aditum precabatur, dicens: Qui sim, unde, cur uenerim, nosti. Scio me non mereri conspectum tuum, tamen nisi uidero, non recedam. Qui bestias suscipis, hominem istiusmodi homo uiuus, magnum populo spectaculum $\mathrm{p}[\mathrm{rae}] \mathrm{buit}$, et postea cadauer ex animae, $\mathrm{n}^{38}$ calore aestatis dissiparetur, sale infusum antiochiam ut ab imperatore uideretur allatum est.

Sed ut propositum persequar, antonius cepta regione ${ }^{39}$ pergebat, ferarum tantum bestias intuens, et heremi latam uastitatem.

Quid ageret, quo uerteret gradum. Iam altera fluxerat dies, restabat unum, ut deseri se a xpo non posse confideret. Pernox secundas in oratione exigit tenebras, et dubia adhuc luce, non procul intuetur lupam sitis ardoribus anhelantem, [f. 190] ad radicem montis irripere ${ }^{40}$.

Quam secutus oculis, et iuxta speluncam cum fera abisset, accedens, caepit introspicere, nichil curiositate proficiente, tenebris arcentibus uisum, uerum ut scriptura ait: Perfecta dilectio foras mittit timorem. Suspenso gradu, et anhelitu ${ }^{41}$ temperato, callidus explorator ingressus est, ac paulatim progrediens sepiusque subsistens, sonum aure ${ }^{42}$ captabat, tandem per caecae noctis horrorem, procul lumen intuitus, dum auidius properat ${ }^{43}$, offenso pede, lapidem instrepitum ${ }^{44}$ concitauit, ac sic beatus paulus ostium quod patebat recludens, sera offirmauit.

Tunc uero antonius pro foribus corruens, usque ad sextam et eo amplius horam, aditum precabatur, dicens: Qui sim, unde, cur uenerim, nosti, scio me non mereri conspectum tuum, tamen nisi uidero non recedam. Qui bestias recipis, hominem

\footnotetext{
38 Certo: ne*.

39 Prima manus correxit. Lectio ante correctionem: religione.

40 Secunda manus in irrepere correxit.

41 U tertia manu super rasuram.

42 Certo: au**re. Secunda manus in margine dextero aure scripsit.

43 At secunda manu, ut videtur, super rasuram.

44 M tertia manu super rasuram, ut videtur.
} 
cur repellis? Quaesiui, et inueni, pulso ut aperiatur; quod si non impetro, hic, hic moriar ante postes tuos: certe sepelies uel cadauer.

Talia perstabat memorans fixusque manebat. Atque huic responsum paucis ita reddidit heros. Nemo sic petit ut minetur, nemo cum lacrimis calumniam facit, et miraris si non recipiam, cum moriturus adueneris?

Sic adridens patefecit ingressum. Quo aperto, dum in mutuos miscentur amplexus, propriis se salutauere nominibus; gratiae Domino in commune referuntur. Et post sanctum osculum residens Paulus cum Antonio, ita exorsus est: En quem tanto labore quaesisti, putribus senectute membris operit inculta canities.

En uides hominem, puluerem mox futurum. Verum quia caritas omnia sustinet, narra mihi, quaeso, ut sese habeat hominum genus. An in antiquis urbibus noua tecta consurgant, quo mundus regatur imperio, an supersint aliqui qui daemonum errore rapiantur. Inter has sermocinationes suspiciunt alitem coruum in ramo arboris consedisse, qui inde leuiter subuolans, integrum panem ante mirantium ora deposuit. Post cuius abscessum: Eia, inquit Paulus, Dominus nobis prandium misit, uere pius, uere misericors. Sexaginta iam anni sunt quod dimidii semper panis fragmen accipio; uerum ad aduentum tuum, militibus suis Christus duplicauit annonam. Igitur in Deum gratiarum actione celebrata, super uitrei marginem fontis uterque consedit. Hic uero quis frangeret panem oborta cur repellis? quaesiui et inueni, pulso ut aperiatur $\mathbf{m}[\mathbf{i h}] \mathbf{i}$; quod si non impetro, hic hic moriar ante postes tuos: certe sepelies uel cadauer mevm ${ }^{45}$.

Talia perstabat memorans, fixusque manebat, ad $\mathbf{q}[\mathbf{u a e}]^{46}$ responsum paucis, ita reddidit heros. Nemo sic petit, ut minetur, nemo cum lacrimis calumniam facit, et miraris si non recipiam, cum moriturus adueneris?

Sic adridens patefecit ingressum. Quo aperto, dum in mutuo miscentur amplexus, propriis se salutauere nominibus; gratiae domino in communae referuntur.

Et ut post sanctum osculum, residens paulus cum antonio, ita exorsus est: En quem tanto labore quesisti, putribus senectute membris, op [er] $\mathrm{it}^{47}$ inculta canities.

En uides ${ }^{48}$ hominem, puluerem mox futurum. Verum quia caritas omnia sustinet, narra mihi, queso, ut sese habeat hominum genus. An in antiquis urbibus, noua tecta consurgant, quo mundus regatur imperio? An supersint aliqui, qui daemonum errore rapiantur? Inter has sermocinationes suspiciunt ${ }^{49}$ alitem coruum, in ramo arboris consedisse, qui [f. $190 \mathrm{v}]$ inde leuiter subuolans, integrum panem ante mirantium ora de posuit.

Post cuius abscessum: eia inquit paulus, Dominus nobis prandium misit, Vere pius, uere misericors. Sexaginta iam anni sunt, quod dimidii semper panis fragmen accipio; uerum ad aduentum tuum, militibus suis, xps duplicauit annonam. Igitur domini $^{50}$ gratiarum actione celebrata, super uitrei marginem fontis uterque consedit. Hic uero quis frangeret panem, oborto ${ }^{51}$

\footnotetext{
45 Litterae evm secunda manus correxit. Super primam m rasura videtur.

$46 \mathrm{D}$ et q secunda manu super rasuram. Secunda manus quę in margine dextero

47 P secunda manu super rasuram.

48 Certo: uide*s. s secunda manu super rasuram.

49 Litterae pici secunda manu super rasuram scriptae sunt.

50 Certo: **d[omi]ni.
} scripsit. 
contentio, pene diem duxit in uesperam. Paulus more cogebat hospitii, Antonius iure refellebat aetatis. Tandem consilium fuit, ut adprehenso e regione pane, dum ad se quisque nititur, pars sua remaneret in manibus. Dehinc paululum aquae prono in fonte ore libarunt, et immolantes Deo sacrificium laudis, noctem transegere uigiliis. Cumque iam esset terris redditus dies, beatus Paulus ad Antonium sic locutus est: Olim te, frater, in istis regionibus habitare sciebam. Olim conseruum meum mihi promiserat Dominus; sed quia iam dormitionis tempus aduenit, et quod semper cupieram dissolui, et esse cum Christo, peracto cursu superest mihi corona iustitiae; tu missus a Domino es, qui humo corpusculum tegas, immo terram terrae reddas. His Antonius auditis, flens et gemens, ne se desereret, atque ut comitem talis itineris acciperet, precabatur. At ille: Non debes, inquit, quaerere quae tua sunt, sed quae aliena. Expedit quidem tibi, sarcina carnis abiecta, Agnum sequi. Sed et caeteris expedit fratribus, ut tuo adhuc instituantur exemplo.

Quamobrem, perge, quaeso, nisi molestum est: et pallium quod tibi Athanasius episcopus dedit, ad obuoluendum corpusculum meum, defer.

Hoc autem beatus Paulus rogauit, non quod magnopere curaret, utrum tectum putresceret cadauer, an nudum (quippe qui tanti temporis spatio, contextis palmarum foliis uestiebatur), sed ut a se recedenti moeror suae mortis leuaretur.

Stupefactus ergo Antonius, quod de Athanasio et pallio eius audierat, quasi Christum in Paulo uidens, et in pectore contentio, pene diem duxit ad uesperam, paulus more cogebat hospitii, antonius iure referebat aetatis. Tandem consilium fuit, ut apprehenso e regione pane, dum ad se quisque nititur, pars sua remaneret in manibus. Dehinc paululum aquae, prono in fonte more libarunt, et immolantes deo sacrificium laudis, noctem transege$\mathrm{re}^{52}$ uigiliis. Cumque iam esset terris redditus dies, beatus paulus ad antonium sic locutus est: Olim te frater, in istis regionibus habitare sciebam, olim conseruum meum mihi promiserat dominus. Sed quia iam dormitionis tempus aduenit, et quod semper cupieram, dissolui et esse cum xo peracto, super est mihi corona iustitiae. Tu missus a domino es, qui humo corpusculum tegas, immo terrae, terram reddas. His antonius auditis, flens et gemens, ne se desereret, atque ut comitem talis itineris acciperet, precabatur. Et ille: non debes inquit querere quae tua sunt, sed quae aliena; expedit quidem tibi sarcina carnis abiecta, agnum sequi, sed et caeteris expedit fratribus, ut tuo adhuc instituantur exemplo.

Quamobrem, perge, queso nisi molestum est: et pallium quod tibi athanasius episcopus dedit, ad obuoluendum corpusculum meum defer.

Hoc autem beatus paulus rogauit, non quod magnopere curaret, utrum tectum putresceret cadauer an nudum, quippe qui tanti temporis spatio, contextis palmarum foliis uestiebatur, sed ut $\mathrm{a}^{53}$ se recedenti meror suae mortis leuaretur ${ }^{54}$.

Stupefactus ergo [f. 191] antonius, quod de anathasio $^{55}$ et pallio eius audierat, quasi in paulo xpm uidens, et in pectore

\footnotetext{
51 Secunda manus tertiam (seu ultimam) o in a correxit.

52 Super primam e secunda manus, super rasuram, i scripsit.

53 A secunda manu, ut videtur, super lineam.

54 Secunda manus in leuiaretur correxit.

55 Secunda manus in margine superiore athanasio scripsit.
} 
eius Deum uenerans, ultra respondere nihil ausus est, sed cum silentio lacrimans, exosculatis eius oculis manibusque, ad monasterium quod postea a Saracenis occupatum est, regrediebatur. Neque uero gressus sequebantur animum, sed cum corpus inane ieiuniis seniles etiam anni frangerent, animo uincebat aetatem.

Tandem defatigatus et anhelus ad habitaculum suum confecto itinere peruenit. Cui cum duo discipuli, qui ei iam longaeuo ministrare coeperant, occurrissent dicentes: Vbi tamdiu moratus es, pater? respondit: Vae mihi peccatori, qui falsum monachi nomen fero. Vidi Eliam, uidi Ioannem in deserto, et uere in paradiso Paulum uidi.

Ac sic ore compresso, et manu uerberans pectus, e cella pallium protulit. Rogantibusque discipulis, ut plenius quidnam rei esset exponeret, ait: Tempus loquendi et tempus tacendi. Tunc egressus foras, et ne modicum quidem cibi sumens, per uiam qua uenerat, regrediebatur, illum sitiens, illum uidere desiderans, illum oculis ac mente complectens. Timebat enim, quod euenit, ne se absente, debitum Christo spiritum redderet. Cumque iam dies inluxisset alia, et trium horarum spatio iter remaneret, uidet inter angelorum cateruas, inter prophetarum et apostolorum choros, niueo Paulum candore fulgentem in sublime conscendere. Et statim in faciem procidens, sabulum capiti superiaciebat, plorans atque eiulans: eius deum uenerans, ultra respondere nichil ausus est. Sed cum silentio lacrimans, et $^{56}$ osculatis eius oculis manibusque, ad monasterium quod postea a sarracenis occupatum est, regrediebatur. Neque uero gressus sequebatur ${ }^{57}$ animum, sed cum corpus inane ieiuniis, seniles etiam anni frangerent, animo uincebat aetatem.

Tandem defatigatus ${ }^{58}$ et anelus ${ }^{59}$, ad habitaculum suum confecto itinere peruenit. Cui cum duo discipuli, qui ei iam longeuo ministrare ceperant, occurrissent dicentes: Vbi tamdiu moratus es pater? R[espondit]: Vae mihi peccatori, qui falsum nomen monachi gero. Uidi heliam, uidi ioh[anne] $\mathrm{m}$ in deserto, et uere in paradysum paulum uidi.

Ac sic ore compresso, atque manu uerberans pectus, e cella pallium protulit. Rogantibusque discipulis ut plenius quidnam rei esset exponeret, Ait: Tempus tacenti, et tempus loquendi. Tunc egressus foras, et ne modicum cibi ${ }^{60}$ sumens, per uiam qua uenerat regrediebatur. Illum sitiens, illum uidere desiderans, illum oculis ac mente complectens, timebat enim quod euenit, ne se absente debitum xo spiritum redderet.

Cumque iam dies illuxisset alia, et trium horarum spatio ${ }^{61}$ iter remaneret, uidit inter angelorum cateruas, inter prophetarum et apostolorum choros, niueo paulum candore fulgentem, in sublime conscendere. Et statim in faciem procidens, sabulum capiti super iacebat $^{62}$, plorans atque heiulans:

56 Secunda manus in margine dextero ex scripsit.

57 Secunda manus in sequebantur correxit.

58 Secunda manus de delevit.

59 Secunda manus in anhelus correxit (littera h super lineam scripta est).

60 Ante cibi secunda manus in margine dextero quidem addidit.

61 Certo: spatio*.

62 Certo: super**iacebat. Litterae ia secunda manu super lineam. Secunda manus iacebat in margine dextero scripsit. 
Cur me, Paule, dimittis? Cur abis insalutatus? Tam tarde notus, tam cito recedis? Referebat postea beatus Antonius tanta se uelocitate, quod reliquum erat uiae, cucurrisse, ut ad instar auis peruolaret; nec immerito, nam introgressus speluncam, uidet genibus complicatis, erecta ceruice, extensisque in altum manibus, corpus exanime. Ac primo, et ipse uiuere eum credens, pariter orabat. Postquam uero nulla, ut solebat, suspiria precantis audiuit, in flebile osculum ruens, intellexit quod etiam cadauer sancti Deum, cui omnia uiuunt, officio gestus precaretur.

Igitur obuoluto et prolato foras corpore, psalmis quoque ex Christiana traditione cantatis, contristabatur Antonius, quod sarculum, quo terram foderet, non habebat, fluctuans uario mentis aestu et secum multa reputans: $\mathrm{Si}$ ad monasterium reuertar, quatridui iter est, si hic maneam, nihil ultra proficiam. Moriar ergo, ut dignum est, et iuxta bellatorem tuum, Christe, ruens, extremum halitum fundam.

Talia eo animo uoluente, ecce duo leones ex interioris eremi parte currentes, uolantibus per colla iubis, ferebantur; quibus aspectis primo exhorruit, rursusque ad Deum mentem referens, quasi columbas uideret, mansit intrepidus. Et illi quidem directo cursu ad cadauer beati senis substiterunt, adulantibusque caudis circa eius pedes accubuere, fremitu ingenti rugientes, prorsus ut intellegeres eos plangere, quo modo poterant. Deinde haud procul coe-
Cur me paule dimittis? cur abis ${ }^{63}$ insalutatus? Tam tarde notus, tam cito recedis? Referebat postea beatus antonius, tanta se uelocitate quod reliquum erat uiae cucurrisse, ut ad instar auis peruolaret. $\mathrm{Nec}$ immerito. Nam introgressus speluncam, uidit genibus complicatis, erecta ceruice, extensisque [f. $191 \mathrm{v}$ ] in altum manibus, corpus exanimae. Ac primo et ipse uiuere eum credens, pariter orabat. Postquam uero nulla ${ }^{64}$ ut solebat suspiria ${ }^{65}$ precantis audiuit, in flebile osculum ruens, intellexit quod etiam cadauer sancti, deum cui omnia uiuunt, officii gestu precaretur.

Igitur obuoluto, et prolato foras corpore, psalmis quoque ex christiana traditione cantatis, contristabatur antonius, quod sarculum, quo terram foderet non habebat. Fluctuans uario mentis aestu, et secum multa reputans: si ad monasterium reuertar, quatridui iter est, si hic maneam, nichil ultra proficiam. Moriar ergo ut dignum est, et iuxta bellatorem tuum xpe ruens, extremum alitum fundam.

Talia animo uoluente, ecce duo leones, ex interioris heremi parte currentes, uolantibus per colla iubis ferebatur ${ }^{66}$. Quibus aspectis, primum exorruit ${ }^{67}$, rursusque ad deum mentem referens, quasi columbas uideret mansit intrepidus. Et illi quidem directo cursu, iuxta cadauer beati senis substiterunt, adulantibusque caudis, circa eius pedes accubuere, fremitu ingenti rugientes. Prorsus ut intellegeres ${ }^{68}$ eos plangere quo modo poterant. Deinde non longe ${ }^{69}$
63 Certo: ab*is.
64 Super a rasura videtur.
65 Certo: su* ${ }^{*}$ spiria.
66 Secunda manus in ferebantur correxit.
67 Secunda manus in margine sinistro hor scripsit.
68 Secunda manus in intelligeres correxit.
69 Secunda manus in margine sinistro haud procul scripsit. 
perunt humum pedibus scalpere, harenamque certatim egerentes, unius hominis capacem locum effodere. Et statim quasi mercedem pro opere postulaturi, cum motu aurium, ceruice deiecta, ad Antonium perrexerunt, manus eius pedesque lingentes, ut ille animaduerteret benedictionem eos a se deprecari. Nec mora, et in laudationem Christi effusus, quod muta quoque animalia sentirent, ait: Domine, sine cuius nutu nec folium arboris defluit, nec unus passerum ad terram cadit, da illis sicut tu scis. Et manu annuens, ut abirent, imperauit. Cumque illi recessissent, sancti corporis onere seniles curuauit humeros, et deposito eo effossam, desuper humum congregans, tumulum ex more conposuit.

Postquam autem dies inluxerat alia, ne quid pius heres ex intestati bonis non possideret, tunicam sibi eius uindicauit, quam in sportarum modum de palmae foliis sibi ipse texuerat, ac sic ad monasterium reuersus, discipulis ex ordine cuncta replicauit;

diebusque solemnibus Paschae uel Pentecostes semper Pauli tunica uestitus est.

Libet in fine opusculi interrogare eos qui patrimonia sua ignorant, qui domos marmoribus uestiunt, qui uno lino uillarum insuunt pretia: huic seni nudo quid umquam defuerit? Vos gemma bibitis, ille concauis manibus naturae satisfecit. Vos in tunicis aurum texitis, ille ne uilissimi quidem mancipii uestri indumentum habuit. caeperunt humum pedibus cauare, harenamque certatim egerentes, unius hominis capacem, locum effodere. Et statim quasi mercedem pro opere postulaturi, cum motu aurium, ceruice deiecta, ad antonium perrexerunt, manus eius pedesque lingentes, ut ille animaduerteret, benedictionem eos a se deprecari. Nec mora, et in laudationem xi effusus, quod muta quoque animalia sentirent, Ait: Domine sine cuius nutu, nec folium arboris defluit, nec unum passerum ad terram cadit, Da illis sicut tu scis.

Et manu annuens, ut abirent imperauit. $\mathrm{Cu}-$ mque illi recessissent, sancti corporis Honeri $^{70}$, seniles curuauit humeros, et deposito eo in fossam, desuper humum congregans, [f. 192] tumulum ex more conposuit. Postquam autem dies illuxerat alia, ne quid pius heres ex intestatis bonis non possideret, tunicam sibi eius uindicauit ${ }^{71}$, quam in sportarum modum de palmae foliis sibi ipse texuerat, ac sic ad monasterium reuersus, discipulis ex ordine cuncta replicauit.

Diebus quoque solempnibus paschae uel pentecostes, semper pauli tunicam uestitus est. Finit uita beatissimi pauli her[e]m[itae]. Libet $^{72}$ in fine opusculi interrogare eos, qui patrimonia sua ignorant, qui domos marmoribus uestiunt, qui uno filo uillarum insuunt $\mathbf{p}[\mathbf{r e}] \mathbf{d i}$ $^{73}$ : huic seni nudo quid unquam defuerit? Vos gemma bibitis, ille concauis manibus naturae satis fecit. Vos in tunicis auro ${ }^{74}$ texitis ille ne ${ }^{75}$ uilissimi quidem mancipii uestri indumenta habuit $^{76}$.

\footnotetext{
70 Secunda manus, ut videtur, $\mathrm{H}$ delevit.

71 Secunda manus in uendicauit correxit.

72 B prima manu, ut videtur, super rasuram.

73 - super a secunda manus, ut videtur, delevit.

74 Secunda manus in aurum correxit.

75 Secunda manus in nec correxit.

76 Certo: $\mathrm{ab}^{*}$ it. Secunda manus $\mathrm{h}$ ante $\mathrm{a}$ addidit et $\mathrm{v}$ post $\mathrm{b}$ super rasuram et lineam scripsit.
} 
Sed e contrario, illi pauperculo paradisus patet, uos auratos gehenna suscipiet. Ille Christi uestem, nudus licet, seruauit; uos uestiti sericis indumentum Christi perdidistis.

Paulus uilissimo puluere opertus iacet resurrecturus in gloriam; uos operosa saxis sepulchra premunt cum uestris opibus arsuros. Parcite, quaeso uos, parcite saltem diuitiis quas amatis. Cur et mortuos uestros auratis obuoluitis uestibus? Cur ambitio inter luctus lacrimasque non cessat? An cadauera diuitum nisi in serico putrescere nesciunt? Obsecro, quicumque haec legis, ut Hieronymi peccatoris memineris: cui si Dominus optionem daret, multo magis eligeret tunicam Pauli cum meritis eius, quam regum purpuras cum regnis suis.
Sed e contrario, illi pauperculo paradysus patet, uos auratos gehenna suscipiet. Ille xi uestem licet nudus seruauit; uos uestiti sericis, indumentum xi perdidistis.

Paulus uilissimo puluere coopertus iacet, resurrecturus iacet in gloria; uos operosa saxis sepulchra premunt, cum uestris opibus arsuros. Parcite queso uos, parcite saltem diuitiis quas amatis. Cur et mortuos uestros auratis obuoluitis uestibus? Cur ambitio inter luctus lacrimasque non cessat? ${ }^{77}$ an cadauera diuitum, nisi in serico, putrescere nesciunt? Obsecro quicumque haec leges, ut hieronimi peccatoris memineris: cui si dominus optionem daret, multo magis eligeret tunicam pauli cum meritis eius, quam regum purpuram cum regnis suis.

$* * *$

Nutriamo una viva speranza che il presente studio contribuisca a sempre migliori scelte per stabilire sia il testo originale della Vita S. Pauli Monachi Thebaei di san Girolamo, sia per far conoscere meglio lo stesso san Paolo di Tebe, e che funga esso stesso, in futuro, come efficace strumento per la classificazione degli eventuali nuovi manoscritti che contengano la Vita del Primo Eremita di Tebe, e che forse saranno un giorno ritrovati dai codicologi, paleografi o studiosi delle antichità cristiane.

\section{The Vita S. Pauli Monachi Thebaei of St. Jerome in the Manuscript Code Vaticanus Latinus 13008}

(summary)

The essay analyzes the text of the Vita S. Pauli Monachi Thebaei of St. Jerome contained in the Manuscript Code Vaticanus Latinus 13008, kept in the Apostolic Vatican Library. In particular, are indicated and highlighted all variations of the Manuscript differing from the text of the critical edition of this work by St. Jerome (see B. Degórski, Edizione critica della "Vita Sancti Pauli Primi Eremitae" di Girolamo, Institutum Patristicum "Augustinianum", Roma 1987).

Keywords: Saint Jerome; Vita S. Pauli Monachi Thebaei; Codicology; Palaeography

77 T prima manu, ut videtur, super rasuram. 


\title{
Vita S. Pauli Monachi Thebaei św. Hieronima znajdująca się w rękopiśmiennym kodeksie Vaticanus Latinus 13008
}

\author{
(streszczenie)
}

Artykuł przedstawia tekst Vita S. Pauli Monachi Thebaei św. Hieronima, który to tekst znajduje się w rękopiśmiennym kodeksie Vaticanus Latinus 13008 przechowywanym w Bibliotece Watykańskiej. W artykule wskazano i zaznaczono wszystkie warianty rękopisu, które różnią się od tekstu wydania krytycznego tego Hieronimowego dzieła (por. B. Degórski, Edizione critica della ,Vita Sancti Pauli Primi Eremitae” di Girolamo, Institutum Patristicum “Augustinianum”, Roma 1987).

Słowa kluczowe: Św. Hieronim; Vita S. Pauli Monachi Thebaei; kodykologia; paleografia

\section{Bibliografia}

\section{Fonti}

Il codice manoscritto Vaticanus Latinus 13008, conservato presso la Biblioteca Apostolica Vaticana.

Hieronymus, Vita S. Pauli Primi Eremitae, ed. B. Degórski, in: Hieronymi historica et hagiographica. Vita Beati Pauli monachi Thebaei. Vita Hilarionis. Vita Malchi monachi captivi. Epistula praefatoria in Chronicis Eusebii Caesariensis. Chronicorum Eusebii Caesariensis continuatio. De viris inlustribus. In Regulae S. Pachomii versionem praefatio (=Girolamo. Opere storiche e agiografiche. Vita di san Paolo, eremita di Tebe. Vita di Ilarione. Vita di Malco, l'eremita prigioniero. Prefazione alla traduzione delle Cronache di Eusebio di Cesarea. Continuazione delle Cronache di Eusebio di Cesarea. Gli uomini illustri. Prefazione alla traduzione della Regola di Pacomio, Hieronymi opera 15), Opere di Girolamo 15, Roma 2014, p. 72-115.

\section{Studi}

Cherf J.F., The Latin Manuscript Tradition of the "Vita Sancti Pauli", in: Studies in the Text Tradition of. St. Jerome's “Vitae Patrum”, ed. W.A. Oldfather, Urbana 1943, p. 133-142.

Degórski B., Edizione critica della "Vita Sancti Pauli Primi Eremitae” di Girolamo, Institutum Patristicum “Augustinianum”, Roma 1987.

Degórski B., Przekaz łacińskich rękopisów Vita Sancti Pauli Primi Eremitae św. Hieronima, Lublin 2000.

Degórski B., I manoscritti della Vita Sancti Pauli Primi Eremitae di san Girolamo conservati nella Biblioteca Apostolica Vaticana, Lublin 2005. 
Degórski B., I manoscritti della Vita Sancti Pauli Primi Eremitae di San Girolamo, conservati nelle biblioteche di Roma, che contengono testo incompleto dell'opera geronimiana o che si ispirano ad essa, Lublin 2011.

Lage Cotos M.E., Sobre los “abusiva” de Huesca, "Studi medievali” 36 (1995) p. 929944.

Leclercq J., S. Antoine dans la tradition monastique médiévale, "Studia Anselmiana" 38 (1956) p. 229-247.

Poncelet A., Catalogus codicum hagiographicorum Latinorum Bibliothecae Vaticanae, Subsidia hagiographica 11, Bruxelles 1910. 